\title{
Improved Muscle Activity Following Motor Training Without Vision of the Throwing Motion in Baseball Pitchers
}

\author{
Won-Ho Choi ${ }^{1}$ and Yun-A Shin ${ }^{2 *}$ \\ ${ }^{1}$ Department of Physical Education, Dankook University, South Korea \\ ${ }^{2}$ Department of Prescription \& Rehabilitation of Exercise College of Physical Exercise, Dankook University, South Korea
}

*Corresponding author: Yun-A Shin, Department of Prescription \& Rehabilitation of Exercise College of Physical Exercise, Dankook University, Republic of Korea, South Korea

To Cite This Article: Yun-A Shin. Improved Muscle Activity Following Motor Training Without Vision of the Throwing Motion in Baseball Pitchers. Am J Biomed Sci \& Res. 2019 - 3(5). AJBSR.MS.ID.000703. DOI: 10.34297/AJBSR.2019.03.000703

Received: June 24, 2019 | Published: July 02, 2019

\begin{abstract}
Throwing motion without vision has the potential as a training method to improve proprioception by concentrating more on joint movements in throwing. Therefore, the purpose of this study was to suggest a method of training motor sense, which can improve ball velocity and the pitchers' control (target accuracy). The study population consisted of $20 \mathrm{high} \mathrm{school} \mathrm{and} \mathrm{college} \mathrm{baseball} \mathrm{players.} \mathrm{Rotator} \mathrm{cuff} \mathrm{and} \mathrm{elbow} \mathrm{joint} \mathrm{muscle} \mathrm{activation,}$ ball velocity, and ball accuracy in the stride, arm cocking, acceleration, and deceleration phases during throwing the ball with maximum effort (TBME) with or without vision were measured. Muscle activation during TBME was shown in the arm cocking and acceleration phases after visual blockage training. However, there were no significant differences in the surface-detected motor unit potential (SMUP), ball velocity, and accuracy when pitching with or without vision and after visual blockage training. Although the significance of this study was low, the SMUP and muscle activity tended to increase with acute practice, which is considered to be a meaningful result. We suggest that further studies should implement the training program for longer periods, wherein adaptation of the nervous system can be achieved through continuous practice.
\end{abstract}

Keywords: Proprioception; Surface-detected motor unit potential; Muscle activity; College baseball pitchers

Abbrevations: EMG: Electromyography; MVIC: Maximal Voluntary Isometric Contraction; SMUP: Surface-Detected Motor Unit Potential; TBME: Throwing the Ball with Maximum Effort

\section{Introduction}

In a baseball game, pitchers play an important role in determining the outcome of the game, and the pitching speed and accuracy are considered highly important factors [1]. However, pitching performance is not easily learned. The throwing technique does not simply involve the use of the hands and arms but is performed by whole body exercises that transmit energy to the distal wrists and fingers through the momentum of the lower limbs and the rotation of the torso and hip [2]. The pitcher needs technical skills to be able to appropriately select and apply various kinds of quick information that is present in a competitive situation in the given environment. The nervous system rapidly performs a goaldirected action, so it should be considered in training to improve performance [3]. While many sensory organs provide information during movement, the selection for the performance of motion takes priority over the acquisition and selection of information through visual attention [4]. Moreover, visual information plays a role in moving the limb in the target direction quickly through the timescale with the dorsal visual stream, thereby reducing the error in performing the motion $[5,6]$.

Therefore, visual attention has been suggested to play an important role in the performance of sports skills [7].

However, the visual information about the goal, that is, the set pitcher's sight on the catcher, is made by the visual system in pitching, but it is different because the role of the visual system in this process is in the spatial aspect, which is the distance to the catcher and various kinds of information such as ball repertory in throwing. Precisely performing the throwing motion requires continuous processing of information about the range of motion, intensity, and forces for successive throwing [2], that is, from the windup, to the stride, acceleration, and deceleration all in a row [8]; this performance information provides proprioception that 
gives information about the position and state of the joint. Accurate and fast pitching are planned based on visual and proprioceptive sensory information, which controls the coordination of body segments and movements through learning and practice. Changes in the kinematic variables such as joint angles and joint angular velocity in performing movements can be learned by kinesthesis and memory $[9,10]$.

In a recent golf putting practice, relying on visual information may have interfered with efficient processing of proprioception due to the superiority of the visual system to other sensory systems. Therefore, it was suggested that training without vision could improve proprioception sensitivity [11]. However, overemphasis on internal attention to body movements (sensory consequences of the motion performed) may interfere with appropriate external attention to the situation and environment through visual interception $[12,13]$. Various training methods have been proposed to improve the information processing ability of proprioception and maintain the optimum attention pattern for the memorized target position and distance [14-17]. However, there is a difference between playing golf and throwing. Golf putting involves watching the ball with the distance to the hole in mind, but in throwing, the sight is concentrated on the target (catcher), and the throwing motion is performed by the integration of muscles and senses such as the movement of the joint, position, force, velocity, and load [18]. In other words, the proprioceptive sense, which plays a role in the internal coordinate system by creating the motor program for planning the movement and performing the exercise properly, provides information about the position and motion of the arm and hand to the body that is critical when throwing the ball in the direction of the visual target. Thus, information on the kinesthetic sense provided by proprioception is very important [19].

Therefore, we considered that blocking vision during the throwing motion could be a training method to improve proprioception by concentrating more on the movement of the joints in throwing. A previous study pertaining to this notion was conducted by analyzing the throwing motion without vision or interception and improving the predictive action outcomes when throwing in the dark $[20,21]$. In particular, if the proprioceptive sensation is activated in the state of non-vision, more motor units may be used to perform the throwing motion. However, few studies have shown that the recruitment of the motor unit and muscle activity are changed according to the adaption of the nervous system. Therefore, the purpose of this study was to suggest a method of training motor senses that can improve the ball speed and control of pitchers effectively. For this purpose, we investigated the effect of training without vision on proprioception.

\section{Materials and Methods}

\section{Study Population}

The study population consisted of 20 pitchers from "J" High School in Gyeonggi Province and "D" College in South Chung cheong Province. All pitchers were high school and university students with at least 7 years of pitching experience and overhand pitchers with no history of elbow or shoulder injury. The physical characteristics of the subjects are shown in Table 1.

\begin{tabular}{l} 
Table 1: Subject characteristics. \\
\hline Position
\end{tabular}

Note: Data are means \pm SD.

\section{Muscle Activation Test}

Electromyography (EMG)electrode attachment: Muscle activation during the exercise load test was measured using a wireless EMG device. The skin was cleaned using alcohol to remove any foreign substances prior to attachment of a total of seven electrodes (distance between centers, $1.5 \mathrm{~cm}$ ). Subsequently, the electrodes were attached to rotator cuff muscles (supraspinatus [SPR], infraspinatus [IFR], and teres minor [TM]) and elbow joint muscles (biceps brachii $[\mathrm{BB}]$, triceps brachii [TB], flexor carpi radialis [FCR], and extensor carpi radialis [ECR]) on the dominant side (right side). Among the rotator cuff muscles, the subscapularis was not measured because the EMG was not supported. Prior to the experiment, each subject underwent practice for measuring the maximal voluntary isometric contraction (MVIC) in each of the eight muscles and was provided adequate rest prior to the actual measurement. The electrode attachment locations on the muscles and MVIC measurement followed the guidelines presented in previous studies [22] and the EMG manufacturer's protocol (SENIAM guidelines). )(Figure 1).

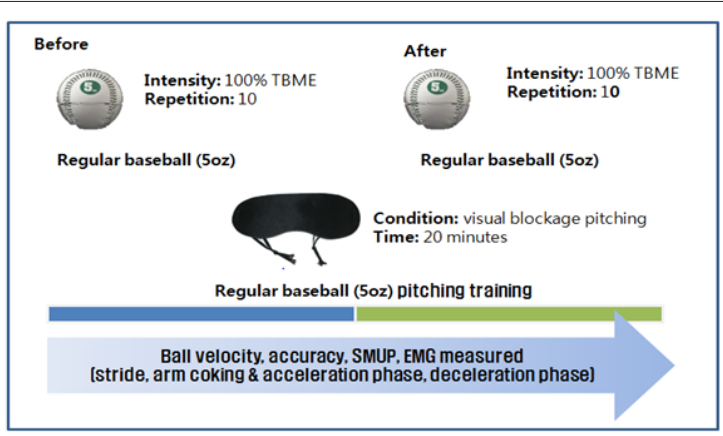

Figure 1: Experimental design. TBME, maximum ball throwing effort; SMUP, surface-detected motor unit potential; EMG, electromyography 
Maximal voluntary isometric contraction measurement: In the MVIC measurement, data were collected by having the specified eight muscles perform a specific motion for $5 \mathrm{~s}$. The SPR muscles was measured by lowering the arm downward and having the shoulder joint exert maximum force toward the ear. The IFR muscle was measured by exerting maximum force while the shoulder performed external rotation toward the outer direction. The TM muscle was measured by erecting the upper body in a straight line and exerting maximum force with the elbow extended to the side in a $90^{\circ}$ direction. The BB muscle was measured with the elbow maintaining a $120^{\circ}$ angle while the forearm exerted maximum force toward the upper arm. The TB muscle was measured with the elbow maintaining a $90^{\circ}$ angle while the forearm exerted maximum force in the direction opposite the upper arm. The FCR muscle was measured with the palm facing anteriorly and the radiocarpal joint pointing posteriorly approximately $30^{\circ}$ toward the back of the hand. The force was exerted with the palm of the hand, and the ECR muscle was measured in the opposite direction.

Surface-detected motor unit potential (SMUP) and muscle activation test during pitching:

The pitchers prepared for throwing the ball with maximum effort (TBME) by performing warm up (WU) and toss-and-catch exercises for 10-15 min. After performing 10 TBME, training for visually blocked throwing was conducted for $20 \mathrm{~min}$. For this, an experienced pitching coach and former professional pitcher for approximately 15 years provided throwing motion training to enable the pitcher's motor program to memorize the pattern by verbal instructions and grasp the pattern of pitching by hand. The coach also adjusted the body position and motion of the visually blocked pitchers so that the ball could be thrown to the catcher's position. After the training, 20 throwing motions and 10 pitches were performed. There was a 10-min break before and after the visually blocked training, considering the game of baseball's offence and defense time (average 5-10 min) during competition. Muscle activation was measured during TBME before and after visual blocking vision. The bandwidth of the EMG signals was filtered using a high-pass filter at $10 \mathrm{~Hz}$ and a low-pass filter at $350 \mathrm{~Hz}$, followed by full-wave rectification. To synchronize the EMG data, 6-mm high-speed digital video cameras were installed to acquire images of the pitching motions, and the images and EMG data were synchronized and analyzed. The baseball pitching motion was divided into six phases for video analysis. In the analysis of muscle activation, only the three phases where the shoulder muscles are highly recruited were selected and analyzed; these included the stride, arm cocking, and arm acceleration phases.

Pitch velocity measurement: In the measurement of the pitch velocity, each subject performed adequate WU (20 min) and tossand-catch exercises ( $\geq 20$ balls). Subsequently, each subject pitched from the mound to the home plate (distance of $18.44 \mathrm{~m}$ ), and a radar gun (Sport Radar, 24.7 GHZ, SP78585, Applied Concepts, Inc., Northbrook, IL, USA) was used to measure the velocity of fastballs that were thrown for strikes.

\section{Statistical Analysis}

The study data were analyzed using SPSS 18.0 for Windows (SPSS Inc., Chicago, IL, USA). All measured values were expressed as means and standard deviations. A paired t-test was used to evaluate the ball speed and number of strikes before and after blocking vision. A repeated measures ANOVA was used to analyze the changes in the SMUP and muscle activation with or without vision, while the least significant difference method was used in the posthoc analysis. The statistical significance level was set at $\mathrm{a}=0.05$.

Results

\section{Changes inball velocity and accuracy with or without vision}

Changes in the ball velocity and accuracy with or without blocking vision are shown in Table 2 . There was no statistically significant difference in ball speed and pitching accuracy after blocking vision compared to before.

Table 2: Changes in ball speed and accuracy with or without vision.

\begin{tabular}{|c|c|c|c|c|}
\hline Condition & TBME-Pre & TBME-Post & t & Sig \\
\hline Ball velocity $(\mathrm{km} / \mathrm{h})$ & $125.20 \pm 4.61$ & $125.30 \pm 5.03$ & -.246 & .811 \\
\hline Ball accuracy (number) & $5.36 \pm 1.01$ & $6.07 \pm 1.07$ & -1.816 & .081 \\
\hline
\end{tabular}

Note: Data are means \pm SD. TBME, throwing ball maximal effort.

\section{Difference in surface-detected motor unit potential with or without vision}

Table 3: SMUP with or without vision (uV, \%MVIC).

\begin{tabular}{|c|c|c|c|c|c|c|c|}
\hline Pitching & SPR & IFR & TM & BB & LB & FCR & ECR \\
\hline TBME-Pre & $111.99 \pm 18.49$ & $77.14 \pm 17.21$ & $66.19 \pm 23.70$ & $33.59 \pm 6.48$ & $38.77 \pm 12.27$ & $40.93 \pm 15.75$ & $69.56 \pm 24.84$ \\
\hline TBME-Post & $129.25 \pm 26.55$ & $101.94 \pm 35.39$ & $63.49 \pm 20.29$ & $46.75 \pm 12.87$ & $62.97 \pm 20.29$ & $66.12 \pm 23.09$ & $47.12 \pm 11.15$ \\
\hline $\mathrm{t}$ & 1.162 & 3.938 & .116 & 3.199 & 3.048 & 1.485 & 1.005 \\
\hline Sig & .299 & .067 & .738 & .095 & .103 & .243 & .333 \\
\hline
\end{tabular}

Note: SPR, supraspinatus; IFR, infraspinatus; TM, teres minor; BB, bicephalus brachii; TB, triceps brachii; FCR, flexor carpi radialis; ECR, extensor carpi radialis; TBME, throwing ball maximal effort. a. Difference between TBME-before and after blocking vision. b. Difference between TBME-before and TBME-after. c. Difference between blocking vision and TBME-after. ${ }^{*} \mathrm{P}<.05 ;{ }^{* *} \mathrm{P}<.01 ;+$, the trend in the difference. 
Changes in the SMUP with or without blocking vision and before and after TBMEare shown in Table 3. Although the SMUP after blocking vision in TBME showed a high level compared to that before blocking vision, except for those in the TM and ECR muscles, there was no statistically significant difference in the results.

\section{Changes in muscle activity with or without vision}

Changes in muscle activity with or without blocking vision and before and after TBME are shown in Table 4. In the stride phase, the SPR muscle activity showed a significant difference $(\mathrm{P}<0.05)$. The results of the posttest showed that the SPR muscle showed higher activity after visual blockage training compared to that before visual blockage training $(\mathrm{P}<0.05)$. In the arm cocking phase, muscle activity of the IFR $(\mathrm{P}<0.05), \mathrm{BB}(\mathrm{P}<0.05)$, and FCR $(\mathrm{P}<0.01)$ muscles also showed a significant difference. The results of the posttest showed that the IFR muscle had lower activity after visual blockage training compared to that before $(\mathrm{P}<0.05)$ and during visual blockage training $(\mathrm{P}<0.01)$. The $\mathrm{BB}$ muscle showed lower activity during visual blockage training compared to that before visual blockage training $(\mathrm{P}<0.05)$. The FCR muscle showed higher activity before visual blockage training compared to that during visual blockage training, with lower activity compared to that after visual blockage training $(\mathrm{P}<0.01)$. In the acceleration phase, activities of the IFR and TB muscles showed a significant difference $(\mathrm{P}=0.054$ and $\mathrm{P}<0.01$, respectively). The results of the posttest showed that the IFR muscle had a tendency of higher muscle activity after visual blockage training compared to that before visual blockage training $(\mathrm{P}<0.05)$. The TB muscle showed higher activity after visual blockage training compared to that before $(\mathrm{P}<0.05)$ and during $(\mathrm{P}<0.01)$ visual blockage training. In the deceleration phase, there were no significant differences.

Table 4: Muscle activity with or without vision (uV, \%MVIC).

\begin{tabular}{|c|c|c|c|c|c|c|c|c|}
\hline Phase & Pitching condition & SPR & IFR & TM & BB & LB & FCR & ECR \\
\hline \multirow{4}{*}{ Stride } & TBME-Pre & $\begin{array}{l}132.91 \\
\pm 22.15\end{array}$ & $81.4 \pm 36.02$ & $53.27 \pm 14.43$ & $44.59 \pm 16.51$ & $54.99 \pm 16.92$ & $47.24 \pm 15.61$ & $42.28 \pm 13.17$ \\
\hline & Blocking vision & $\begin{array}{l}148.27 \\
\pm 25.36 \\
\end{array}$ & $60.87 \pm 51.03$ & $55.49 \pm 41.63$ & $44.41 \pm 34.66$ & $50.47 \pm 27.98$ & $52.95 \pm 33.42$ & $42.30 \pm 26.33$ \\
\hline & TBME-Post & $\begin{array}{c}155.47 \\
\pm 28.04^{\mathrm{b}^{*}}\end{array}$ & $77.94 \pm 39.39$ & $66.26 \pm 27.60$ & $39.00 \pm 23.14$ & $52.23 \pm 13.67$ & $51.36 \pm 28.42$ & $54.17 \pm 13.10$ \\
\hline & F (Sig) & $9.393(.018)$ & $1.833(.196)$ & $.941(.414)$ & $.409(.672)$ & $.068(.934)$ & $.490(.623)$ & $1.368(.287)$ \\
\hline \multirow{4}{*}{ Cocking } & TBME-Pre & $\begin{array}{l}119.72 \\
\pm 28.17\end{array}$ & $\begin{array}{l}100.62 \\
\pm 29.80\end{array}$ & $67.89 \pm 16.55$ & $46.34 \pm 18.51$ & $50.41 \pm 14.98$ & $46.83 \pm 13.14$ & $\begin{array}{c}57.13 \\
\pm 12.66^{\mathrm{a}^{*}, \mathrm{~b}^{* *}}\end{array}$ \\
\hline & Blocking vision & $\begin{array}{l}109.26 \\
\pm 53.16\end{array}$ & $90.97 \pm 26.99$ & $59.62 \pm 34.72$ & $31.63 \pm 24.66$ & $43.05 \pm 29.78$ & $45.27 \pm 27.57$ & $43.31 \pm 18.76$ \\
\hline & TBME-Post & $\begin{array}{l}124.75 \\
\pm 57.71\end{array}$ & $\begin{array}{c}135.45 \\
\pm 28.93^{b^{*}, c^{* *} a^{*}, c^{* *}}\end{array}$ & $65.52 \pm 24.24$ & $45.33 \pm 13.46^{c^{*}}$ & $58.13 \pm 31.24$ & $48.24 \pm 20.93$ & $67.62 \pm 13.96$ \\
\hline & F (Sig) & $.011(.918)$ & $7.966(.005)$ & $.364(.701)$ & $4.287(.035)$ & $\begin{array}{c}.713 \\
(.507)\end{array}$ & $.349(.711)$ & $\begin{array}{l}10.605 \\
(.002)\end{array}$ \\
\hline \multirow{4}{*}{ Acceleration } & TBME-Pre & $\begin{array}{l}122.13 \\
\pm 32.58\end{array}$ & $\begin{array}{l}132.42 \\
\pm 24.40\end{array}$ & $78.35 \pm 20.78$ & $63.15 \pm 17.31$ & $48.72 \pm 17.22$ & $47.71 \pm 13.31$ & $74.22 \pm 28.84$ \\
\hline & Blocking vision & $\begin{array}{l}118.65 \\
\pm 42.53\end{array}$ & $92.77 \pm 49.92$ & $58.00 \pm 38.21$ & $56.95 \pm 23.08$ & $51.99 \pm 24.07$ & $54.87 \pm 24.95$ & $66.37 \pm 48.46$ \\
\hline & TBME-Post & $\begin{array}{l}116.72 \\
\pm 51.83\end{array}$ & $\begin{array}{c}124.60 \\
\pm 26.16^{c+}\end{array}$ & $83.75 \pm 41.46$ & $69.97 \pm 35.33$ & $\begin{array}{c}82.10 \\
\pm 26.17^{\mathrm{b}^{*}, \mathrm{c}^{* *}}\end{array}$ & $54.88 \pm 16.29$ & $\begin{array}{c}84.14 \\
\pm 25.26^{b^{*}}\end{array}$ \\
\hline & F (Sig) & $.010(.905)$ & $3.617(.054)$ & $1.965(.177)$ & $1.089(.363)$ & $7.907(.005)$ & $1.205(.329)$ & $1.380(.284)$ \\
\hline \multirow{4}{*}{ Deceleration } & TBME-Pre & $80.46 \pm 18.73$ & $75.27 \pm 16.48$ & $80.25 \pm 12.98$ & $42.12 \pm 16.88$ & $43.22 \pm 21.26$ & $48.57 \pm 11.17$ & $58.39 \pm 19.45$ \\
\hline & Blocking vision & $94.54 \pm 41.41$ & $57.94 \pm 20.66$ & $78.82 \pm 37.64$ & $54.24 \pm 31.18$ & $58.59 \pm 41.09$ & $50.32 \pm 17.82$ & $60.00 \pm 60.00$ \\
\hline & TBME-Post & $\begin{array}{l}108.64 \\
\pm 40.72\end{array}$ & $78.25 \pm 50.46$ & $99.64 \pm 47.32$ & $59.36 \pm 24.73$ & $59.57 \pm 25.45$ & $56.23 \pm 13.39$ & $63.85 \pm 63.86$ \\
\hline & F (Sig) & $1.817(.199)$ & $1.128(.351)$ & $1.269(.312)$ & $.967(.404)$ & $1.500(.257)$ & $2.008(.171)$ & $.544(.592)$ \\
\hline
\end{tabular}

praspinatus; IFR, infraspinatus; TM, teres minor; BB, bicephalus brachii; TB, triceps brachii; FCR, flexor carpi radialis; ECR, extensor carpi radialis; TBME, throwing ball maximal effort. a. Difference between TBME-before and after blocking vision. b. Difference between TBME-before and TBME-after. c. Difference between blocking vision and TBME-after. ${ }^{*} \mathrm{P}<.05$; ${ }^{*} \mathrm{P}<.01 ;+$, the trend in the difference.

\section{Discussion}

After examining the effects of visual blocking pitching practice, muscle activity showed a tendency to increase in the arm cocking and acceleration phases. However, there was no difference in ball speed and accuracy. Moreover, the SMUP analyzed by the motor unit showed a higher mean value after visual blockage training than that before visual blockage training during the TBME, but there was no significant difference. Throwing skills are developed through 
repetitive practice and training. However, the motor program improved the sensitivity of kinetic sensation by remembering the difference in position, distance, load, force, etc., as well as the enhancement of physical fitness such as strength and power [23]. The motor program, which determines and controls the activity of the muscles so as to perform the exercise properly, continuously receives information on the motion range, direction, and position from each part of the body during movement to improve and control movement in the initial training stage [20]. There are constant and variable practices in terms of motor learning in order to quickly acquire and conduct these motor programs. Constant practice is performed in one way only, whereas variable practice utilizes various methods in combinations regardless of the order. Previous studies have shown that variable practice is more effective than constant practice [24]. In this study, we tried to adjust the velocity and direction during pitching by intercepting vision as a variable practice method and teaching the direction and pattern of the joint by hand so that the proper movement of the joint could occur. In order to the throw, we calibrated the position of the body and motion by providing verbal instructions. The performed action and efficient motor program were spontaneously modulated and facilitated by informative kinematic cues [25,26]. Ball speed, pitching accuracy, and SMUP during TBME all showed a high level after visual blockage training, but there was no statistical significance relative to before visual blockage training.

In a previous study, it was suggested that presenting a taskspecific exercise might induce the agility, proprioceptive sensory enhancement, muscle strength, and contraction timing and intensity by controlling various joint participation, speed, and direction $[27,28]$. However, in this study, there was no significance because of the relatively acute short practice time, although the average overall SMUP increased. The study results considered the effects and adaptation of the nervous system, because muscle contractions are regulated by the central nervous system and influenced by the fast or slow twitch fibers [29] or neural reinforcement patterns. Furthermore, improvement in muscle function is more successful through mobilization of motor units and is much more effective through the activity of the nervous system, thus providing more muscle power [30-33]. The stride phase is triggered by shoulder elevation and abduction of $80-100^{\circ}$, which is the first time the foot touches the ground, and the deltoid and SPR muscles are activated to maintain abduction and elevation $[34,35]$.

In this study, the activity of the SPR muscle in the stride phase increased after visual block age training. In the arm cocking phase, the posterior triceps, latissimus dorsi, pectoralis minor, and IFR are responsible for external rotation of the shoulder [36]. The flexion of the elbow increases the biceps activity, and the activities of the IFR and BB muscles in this study increased after visual blockage training compared to those before visual blockage training. The acceleration phase is changed from the arm cocking to acceleration phase, and when maximum external rotation is performed, the internal torque is $111 \mathrm{Nm}$, and the highest muscle activity is reported in this phase [37]. In this study, the activity of the IFR and BB muscles increased after visual blockage training compared to that before visual blockage training. The deceleration phase begins to slow down the arm while releasing the ball. The arm continues adduction, but the joint angular velocity decreases (Werner et al., 1993). In this study, there was no significant difference in muscle activity in this phase during and after visual blockage training.

The role of visual and proprioceptive senses depends on the phase of throwing. In the first phase including goal analysis, the visual system acts as the main sense, but in the demonstration and performance phase of intended motion, the proprioceptive sense plays a major role. In other words, vision-dependent pitching can distract attention to the processing of visual information even in areas where the use of visual information is not required, which can adversely affect information processing and interpretation of proprioceptive senses [38]. In learning the exercise function to exert the best performance in sports, it is necessary to learn how to control the coordination of the body segments involved in the performance and the size and timing of the force exerted by the body segment. Sports motions should be performed by processing and interpreting proprioceptive information [39]. Once the action is learned and proficiency is developed by repetitive pitching exercises, the motor program can rapidly be performed independently, since it requires little adjustment and modification of the initial steps [40]. In other words, it controls the muscles of the limbs that move and control the body segments through the proprioceptive senses and improves the processing ability of range and direction of motion through kinesthetic sense, thus making throwing possible even in the dark [41].

Pitching exercises without vision can facilitate information processing and utilization of coherence patterns of body segments, along with the magnitude and timing of the forces exerted. Thus, the sensitivity of proprioception on body segment information can be improved [42]. Therefore, the increase in the activities of the agonist muscles related to the throwing motion performance in each of the phases in this study suggested that this was accompanied by either a concomitant increase in the concentration of vision when the visual information was available after visual blockage training, or an increase in the mobilization ability of the motor unit.

\section{Conclusion}

In this study, we investigated changes in motor unit mobilization, muscle activity, ball speed, and accuracy during each throwing phase before and after visual blockage training. There were no significant differences except for the increase in muscle activity. However, although the significance of this study was low, the SMUP and muscle activity tended to increase with acute practice, which is considered to be a meaningful result. Therefore, we suggest that further studies should implement a training program with a longer period in which adaptation of the nervous system can be achieved through continuous practice.

\section{Acknowledgements}

The authors are grateful to the baseball players who participated in the experiment and D university baseball coach Yoo-Jin Kim who assisted in the experiment. 


\section{Conflict of interest}

The authors declare no conflicts of interest. This study was not financially supported by a granting agency.

\section{References}

1. Yang WW, Liu YC, Lu LC, Chang HY, Chou PPH, et al. (2013) Performance enhancement among adolescent players after 10 weeks of pitching training with appropriate baseball weights. J Strength Cond Res 27: 3245-3251.

2. Stodden DF, Langendorfer SJ, Fleisig GS, Andrews JR (2006) Kinematic constraints associated with the acquisition of overarm throwing part I: step and trunk actions. Res Q Exerc Sport 77(4): 417-427.

3. Urbin MA (2013) Visual regulation of overarm throwing performance. Exp Brain Res 225(4): 535-547.

4. Hagemann N, Schorer J, Cañal-Bruland R, Lotz S, Strauss B (2010) Visual perception in fencing: Do the eye movements of fencers represent their information pickup? Atten Percept Psychophys 72(8): 2204-2214.

5. Elliott D (1992) Intermittent versus continuous control of manual aiming movements. In Proteau L, Elliott D, editors. Vision and motor control. Amsterdam, The Netherlands: Elsevier Science 85: 33-48.

6. Milner AD, Goodale MA (2008) Two visual systems re-viewed. Neuropsychologia 46(3): 774-785.

7. Abernethy B (1990) Anticipation in squash: differences in advance cue utilization between expert and novice players. J Sports Sci 8(1): 17-34.

8. Shin YA, Choi WH (2018) Effects of weighted baseball throwing during warm-up on ball velocity and upper extremity muscle activation in baseball pitchers. J Exerc Rehabil 14(3): 436-444.

9. Anderson DI, Sidaway B (1994) Coordination changes associated with practice of a soccer kick. Res Q Exerc Sport 65(2): 93-99.

10. Steenbergen B, Marteniuk RG, Kalbfleisch LE (1995) Achieving coordination in prehension: joint freezing and postural contributions. J Mot Behav 27(4): 333-348.

11. Touzalin-Chretien P, Ehrler S, Dufour A (2010) Dominance of vision over proprioception on motor programming: evidence from ERP. Cereb Cortex 20(8): 2007-2016.

12. Wulf G, McNevin N, Shea CH (2001) The automaticity of complex motor skill learning as a function of attentional focus. Q J Exp Psychol A 54(4):1143-1154.

13. Wulf G, Shea C, Park JH (2001) Attention and motor performance: preferences for and advantages of an external focus. Res Q Exerc Sport 72(4): 335-344.

14. Bartlett RM, Stockill NP, Elliott BC, Burnett AF (1996) The biomechanics of fast bowling in men's cricket: a review. J Sports Sci 14(5): 403-424.

15. Güldenpenning I, Kunde W, Weigelt M (2017) How to trick your opponent: a review article on deceptive actions in interactive sports. Front Psychol 8: 917.

16. Huys R, Cañal-Bruland R, Hagemann N, Beek PJ, Smeeton NJ, et al. (2009) Global information pickup underpins anticipation of tennis shot direction. J Mot Behav 41(2):158-171.

17. Smeeton NJ, Huys R (2011) Anticipation of tennis-shot direction from whole-body movement: the role of movement amplitude and dynamics. Hum Mov Sci 30(5): 957-965.

18. Haggard P (1992) Chapter 7 Multi-sensory control of coordinated movement. Advances in Psychology 84:195-231.

19. Latash ML, Scholz JP, Schöner G (2002) Motor control strategies revealed in the structure of motor variability. Exerc Sport Sci Rev 30(1): 26-31.
20. Casile A, Giese MA (2006) Nonvisual motor training influences biological motion perception. Curr Biol 16(1): 69-74.

21. Mulligan D, Hodges NJ (2014) Throwing in the dark: improved prediction of action outcomes following motor training without vision of the action. Psychol Res 78(5): 692-704.

22. Cram JR, Kasman GS, Holtz J (1999) Introduction to Surface Electromyography J Athl Train 34(1): 69.

23. Widmaier E, Raff H, Strang K (2010) Vander's human physiology: the mechanisms of body function, $\left(12^{\text {th }}\right.$ edn) McGraw-Hill Korea, South Korea.

24. Schmit RA, Lee TD (2005) Motor control and learning: a behavioral emphasis. Champaign IL, Human Kinetics, USA.

25. Vesper C, Richardson MJ (2014) Strategic communication and behavioral coupling in asymmetric joint action. Exp Brain Res 232(9): 2945-2956.

26. Candidi M, Curioni A, Donnarumma F, Sacheli LM, Pezzulo G (2015) Interactional leader-follower sensorimotor communication strategies during repetitive joint actions. J R Soc Interface 129(110): 0644.

27. Vearrier LA, Langan J, ShumwayCook A, Wollacott M (2005) An intensive massed practice approach to retraining balance poststroke. Gait Posture 22(2):154-163.

28. Johnson MJ, Feng X, Johnson LM, Winters JM (2007) Potential of a suite of robot computer assisted motivating systems for personalized, homebased, stroke rehabilitation. J Neuroengineering Rehabil 4: 6 .

29. Buller AJ, Kean CJ, Ranatunga KW (1987) Transformation of contraction speed in muscle following cross-reinnervation: dependence on muscle size. J Muscle Res Cell Motil 8(6): 504-516.

30. Bandy WD, Lovelace-Chandler V, Mckitrick-Brandy B (1990) Adaptation of skeletal muscle to resistance training. J Orthop Sports Phys Ther 12(6): 248-255.

31. Kraemer WJ, Deschenes MR, Fleck SJ (1988) Physiological adaptations to resistance exercise. Implications for athletic conditioning. Sports Med 6(4): 246-256.

32. Rose SJ, Rothstein JM (1982) Muscle mutability. Part 1. General concepts and adaptations to altered patterns of use. Phys Ther 62(12): 17731787.

33. Hong AR, Hong SM, Shin YA (2014) Effects of resistance training on muscle strength, endurance, and motor unit according to ciliary neurotrophic factor polymorphism in male college students. J Sports Sci Med 13(3): 680-688.

34. Bradley JP, Tibone JE (1991) Electromyographic analysis of muscle action about the shoulder. Clin Sports Med 10(4): 789-805.

35. Meister K (2000) Injuries to the shoulder in the throwing athlete. Part one biomechanics/pathophysiology/classification of injury. Am J Sports Med 28(20): 265-275.

36. Wilk KE, Mesiter K, Fleisig G, Andrews JR (2000) Biomechanics of the overhead throwing motion. Sports Medicine and Arthroscopy Review 8(2): 124-134.

37. Werner SL, Flesig GS, Dillman CJ, Andrews JR (1993) Biomechanics of the elbow during baseball pitching. J Sports Phy Ther 17(6): 274-278.

38. Goodale MA (2008) Action without perception in human vision. Cogn Neuropsychol 25(7-8): 891-919.

39. Studenka BE, Zelaznik HN, Balasubramaniam R (2012) The distinction between tapping and circle drawing with and without tactile feedback: an examination of the sources of timing variance. Q J Exp Psychol (Hove) 65(6): 1086-1100.

40. Knoblich G, Seigerschmidt E, Flach R, Prinz W (2002) Authorship effects in the prediction of handwriting strokes: evidence for action simulation during action perception. Q J Exp Psychol A 55(3): 1027-1046. 
41. Khanmohammadi R, Someh M, Ghafarinejad F (2011) The effect of cryotherapy on the normal ankle joint position sense. Asian J Sports Med 2(2): 91-98.
42. Meacci WG, Pastore DL (1995) Effects of occluded vision and imagery on putting golf balls. Percept Mot Skills 80(1): 179-186. 